Document downloaded from:

http://hdl.handle.net/10251/103764

This paper must be cited as:

Cordero Barbero, A.; Soleymani, F.; Torregrosa Sánchez, JR.; Haghani, FK. (2017). A family of Kurchatov-type methods and its stability. Applied Mathematics and Computation. 294:264-279. doi:10.1016/j.amc.2016.09.021

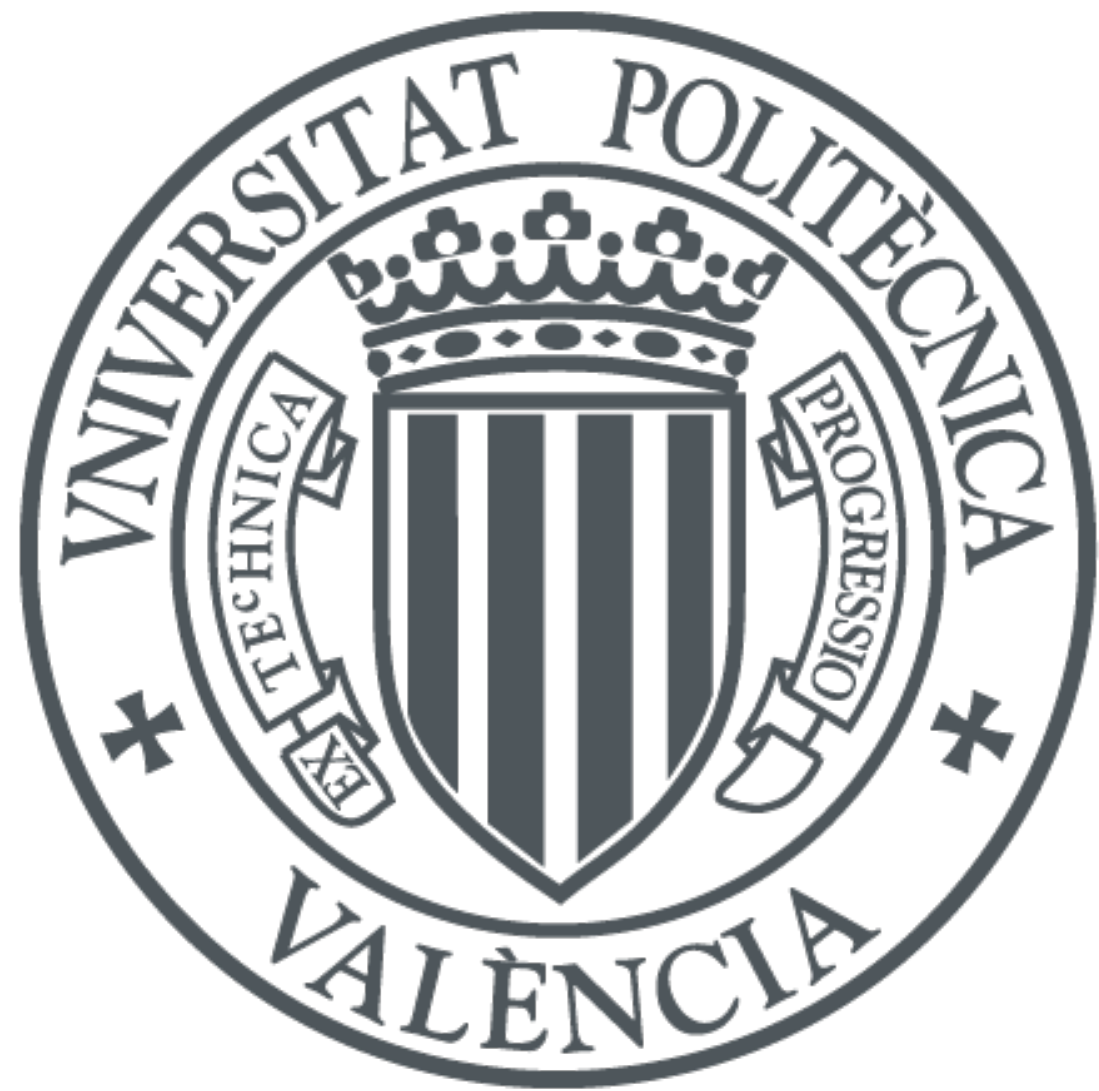

The final publication is available at

http://dx.doi.org/10.1016/j.amc.2016.09.021

Copyright Elsevier

Additional Information 


\title{
A family of Kurchatov-type methods and its stability
}

\author{
A. Cordero ${ }^{a, 1}$, F. Soleymani ${ }^{a, b, 2}$, J.R. Torregrosa ${ }^{a, 3}$, F. Khaksar Haghani ${ }^{b, 4}$ \\ ${ }^{a}$ Instituto Universitario de Matemática Multidisciplinar, Universitat Politècnica de València, 46022 València, Spain \\ ${ }^{b}$ Department of Mathematics, Faculty of Basic Science, Shahrekord Branch, Islamic Azad University, Shahrekord, Iran
}

\begin{abstract}
We present a parametric family of iterative methods with memory for solving of nonlinear problems including Kurchatov's scheme, preserving its second-order of convergence. By using the tools of multidimensional real dynamics, the stability of members of this family is analyzed on low-degree polynomials, showing some elements of this class more stable behavior than the original Kurchatov's method. The iteration is extended for multi-dimensional case. Computational efficiencies of proposed technique is discussed and compared with the existing methods. A couple of numerical examples are considered to test the performance of the new family of iterations.

Keywords: Iterative methods; with memory; R-order; divided difference operator; stability; bifurcation diagrams; chaos.
\end{abstract}

\section{Motivation}

Computing the solution of nonlinear equations by using iterative methods is considered in this work. In general, the solution $\alpha$ of a nonlinear function can be obtained as a fixed point of some function $f: D \subseteq \mathbb{R} \rightarrow \mathbb{R}$ by means of the following fixed-point iteration

$$
x_{k+1}=\phi\left(x_{k}\right), \quad n \geq 0 .
$$

The most widely used method for this purpose is the classical Newton's method and its derivative-free form known as Steffensen's scheme [27]:

$$
x_{k+1}=x_{k}-\left[x_{k}, w_{k}, f\right]^{-1} f\left(x_{k}\right), \quad k \geq 0,
$$

wherein $w_{k}=x_{k}+f\left(x_{k}\right)$. These methods converge quadratically under the conditions that the function $f$ is continuously differentiable and a good initial approximation $x_{0}$ is given, [28].

Here, the first order divided difference operator (DDO) of $f$ on the points $x$ and $y$ can be defined componentto-component as follows [20]:

$$
[x, y ; f]_{i, j}=\frac{f_{i}\left(x_{1}, \ldots, x_{j}, y_{j+1}, \ldots, y_{n}\right)-f_{i}\left(x_{1}, \ldots, x_{j-1}, y_{j}, \ldots, y_{n}\right)}{x_{j}-y_{j}}, \quad 1 \leq i, j \leq n \text {. }
$$

According to the recent trend of researches in this topic, iterative methods with memory (also known as self-accelerating schemes) are worth studying. This is a naming due to Traub [28] when more than one iteration of an scheme, or an updating of a free (non-zero) parameter are applied per cycle so as to calculate the next approximate value(s).

To review the literature briefly, we remark that optimal Steffensen-type families without memory for solving nonlinear equations were introduced in [22] in a general form, two-step self-accelerating Steffensen-type methods and their applications in the solution of nonlinear systems and nonlinear differential equations were discussed in [30]. For further background on this topic, one may consult $[4,21]$.

Due to the fact that (2) with $\beta=1$ suffers of too small local convergence regions [7], two-points method which falls under the definition of schemes with memory due to the definition of [28] are taken into account from time to time. For instance, the secant method is given by

$$
x_{k+1}=x_{k}-\left[x_{k-1}, x_{k}, f\right]^{-1} f\left(x_{k}\right), \quad k \geq 0,
$$

\footnotetext{
${ }^{1}$ Corresponding author. Email: acordero@mat.upv.es.

2 Email: fazlollah.soleymani@gmail.com.

${ }^{3}$ Email: jrtorre@mat.upv.es.

${ }^{4}$ Email: haghani1351@yahoo.com.
} 
with the R-order $\frac{1+\sqrt{5}}{2}$ while the quadratically convergent scheme of Kurchatov is expressed as follows [17]:

$$
x_{k+1}=x_{k}-\left[x_{k-1}, 2 x_{k}-x_{k-1}, f\right]^{-1} f\left(x_{k}\right), \quad k \geq 0 .
$$

Motivated by the recent developments in this area (see, for example, [14]), we here propose a family of parametric methods, variants of (5) and an extension for systems of nonlinear equations, preserving the order of convergence of the original scheme. The stability of the different elements of this family is analyzed on lowdegree polynomials, showing that some members of the class have good stability properties, meanwhile others present chaotic behavior. Hence, this would be our main motivation in this work.

The paper is divided into several sections and is organized as follows. After this introductory review, a family of Kurchatov-type schemes is proposed and studied in Section 2. The drawbacks and strong points are also pointed out. Section 3 includes the analysis of the stability by providing the dynamical behavior of the these schemes. In Section 4, some generalization of the proposed variant for systems of nonlinear equations are brought forward. In Section 5, various numerical examples are considered to confirm the theoretical results. A comparison with the existing methods is also presented in this section. Concluding remarks are given in Section 6.

\section{Methodology and convergence analysis}

One technique to extend the already known schemes and provide some generalizations of them is due to imposing a parameter into their structure so as to keep the convergence R-order and improve it to some extend under some considerations.

Let us re-write the extra point involved in (5) as comes next:

$$
2 x_{k}-x_{k-1}=x_{k}+x_{k}-x_{k-1} .
$$

The increment $x_{k}-x_{k-1}$ would roughly be equal to $f\left(x_{k}\right)$ in the convergence phase. On the other hand, in (2), we have $w_{k}=x_{k}+\beta f\left(x_{k}\right)$, so we could apply a similar idea here for (5) to impose a free nonzero parameter $\beta$ into the structure in what follows:

$$
\left\{\begin{array}{l}
l_{k}=\beta x_{k-1}, \quad w_{k}=x_{k}+\beta\left(x_{k}-x_{k-1}\right), \\
f\left[w_{k}, l_{k}\right]=\frac{f\left(w_{k}\right)-f\left(l_{k}\right)}{w_{k}-l_{k}}, \\
x_{k+1}=x_{k}-\frac{f\left(x_{k}\right)}{f\left[w_{k}, l_{k}\right]}, \quad k=1,2, \ldots
\end{array}\right.
$$

Theorem 1. Let the function $f(x)$ is at least three times differentiable in a neighborhood of its simple zero $\alpha$. If an initial approximations $x_{0}$ and $x_{1}$ are sufficiently close to $\alpha$, then, convergence $R$-order of the improved Kurchatov's method with memory (7) is two.

Proof. The R-quadratic convergence of (7) can be proved with the help of the majorants of Kantorovich (see e.g. [5]). In this paper, we introduce a different procedure to prove the R-quadratic convergence, where we show a shorter alternative analytic proof. To this end, let the sequences $\left\{x_{k}\right\}$ and has convergence order $r$, $e_{k}=x_{k}-\alpha, f(\alpha)=0$ and $c_{j}=\frac{f^{(j)}(\alpha)}{j ! f^{\prime}(\alpha)}, j \geq 2$. Using Taylor expansion, we have

$$
f\left(x_{k}\right)=f^{\prime}(\alpha)\left[e_{k}+c_{2} e_{k}^{2}+c_{3} e_{k}^{3}+c_{4} e_{k}^{4}+c_{5} e_{k}^{5}\right]+O\left(e_{k}^{5}\right),
$$

and

$$
\begin{aligned}
f\left[w_{k}, l_{k}\right]= & \left.\left(f^{\prime}(\alpha)+c_{2} f^{\prime}(\alpha)(1+\beta) e_{k}+c_{3} f^{\prime}(\alpha)(1+\beta]\right)^{2} e_{k}^{2}+O\left(e_{k}^{3}\right)\right), \\
& +\left(-c_{3} f^{\prime}(\alpha) \beta(1+\beta) e_{k}-2\left(c_{4} f^{\prime}(\alpha) \beta(1+\beta)^{2}\right) e_{k}^{2}+O\left(e_{k}^{3}\right)\right) e_{k-1}, \\
& +\left(c_{3} f^{\prime}(\alpha) \beta^{2}+2 c_{4} f^{\prime}(\alpha) \beta^{2}(1+\beta) e_{k}+4 c_{5} f^{\prime}(\alpha) \beta^{2}(1+\beta)^{2} e_{k}^{2}+O\left(e_{k}^{3}\right)\right) e_{k-1}^{2}+O\left(e_{k-1}^{3}\right) .
\end{aligned}
$$


Combining (8) and (9) into (7), one get that

$$
\begin{aligned}
e_{k+1}= & \left(c_{2} \beta e_{k}^{2}+O\left(e_{k}^{3}\right)\right)+\left(-c_{3} \beta(1+\beta) e_{k}^{2}+O\left(e_{k}^{3}\right)\right) e_{k-1} \\
& +\left(c_{3} \beta^{2} e_{k}+\beta^{2}\left(2 c_{4}(1+\beta)-c_{2}\left(c_{3}+2 c_{3} \beta\right)\right) e_{k}^{2}+O\left(e_{k}^{3}\right)\right) e_{k-1}^{2}+O\left(e_{k-1}^{3}\right) .
\end{aligned}
$$

To unify the error equation we extract the coefficients of $e_{k}^{1}$ and $e_{k}^{2}$ and attain the following asymptotical error equation

$$
e_{k+1} \sim \beta^{2} c_{3} e_{k-1}^{2} e_{k}-\beta\left(c_{2}\left(\beta(2 \beta+1) c_{3} e_{k-1}^{2}-1\right)+(\beta+1) e_{k-1}\left(c_{3}-2 \beta c_{4} e_{k-1}\right)\right) e_{k}^{2} .
$$

Note that in general, the error equation should read

$$
e_{k+1} \sim A e_{k}^{p}
$$

where $A$ and $p$ are to be determined. Hence, one has $e_{k} \sim A e_{k-1}^{p}$, and subsequently $e_{k-1} \sim A^{-1 / p} e_{k}^{1 / p}$. Thus, it is easy to obtain

$$
e_{k}^{p} \sim e_{k}^{\frac{2}{p}} e_{k}
$$

which results in the equation

$$
p=1+\frac{2}{p},
$$

with two solutions $\{-1,+2\}$. Clearly the value for $p=+2$ is acceptable and would be the convergence R-order of the family (7) with memory. The proof is complete.

\section{Stability analysis}

The dynamical behavior of the operators associated to numerical methods is an efficient tool for analyzing the stability of the methods. This kind of analysis has been widely developed in recent years on iterative methods without memory, by using the tools of complex discrete dynamics (see, for example, $[3,7,10,19]$ ). However, iterative methods with memory are not eligible to be analyzed by using these techniques and, very recently, in $[6,8]$, the authors focused their qualitative analysis by transforming them into multidimensional dynamical systems, whose dynamical properties could be studied by using the same standard tools as the classical Henon map (see $[2,13])$. In this section, we build the discrete dynamical system associated to $(7)$ in order to carry out its qualitative study.

The expression of any iterative method with memory, which uses two previous iterations to calculate the following estimation, is

$$
x_{k+1}=g\left(x_{k-1}, x_{k}\right), \quad k \geq 1,
$$

where $x_{0}$ and $x_{1}$ are the initial guesses. A fixed point of this method will be obtained when not only $x_{k+1}=x_{k}$, but also $x_{k-1}=x_{k}$. In order to obtain them, we define the fixed point function $G: \mathbb{R}^{2} \longrightarrow \mathbb{R}^{2}$ by means of:

$$
\begin{aligned}
G\left(x_{k-1}, x_{k}\right) & =\left(x_{k}, x_{k+1}\right), \\
& =\left(x_{k}, g\left(x_{k-1}, x_{k}\right)\right), \quad k=1,2, \ldots
\end{aligned}
$$

This definition can be extended in a natural way to adapt it to iterative schemes with memory that use more than two previous iterations per step.

As $\left(x_{k-1}, x_{k}\right)$ is a fixed point of $G$ if

$$
G\left(x_{k-1}, x_{k}\right)=\left(x_{k-1}, x_{k}\right),
$$

then, $x_{k+1}=x_{k}$ and $x_{k-1}=x_{k}$. So, the mentioned conditions are satisfied. 
Now, we define a discrete dynamical system in the plane from the function $G: \mathbb{R}^{2} \rightarrow \mathbb{R}^{2}$, given by

$$
\begin{aligned}
G(z, x) & =(x, g(z, x)) \\
& =\left(x, x+\frac{f(x)(x+\beta x-2 \beta z)}{f(\beta z)-f((\beta+1) x-\beta z)}\right)
\end{aligned}
$$

where $g$ is the operator of the iterative method with memory and $f$ is the nonlinear function whose root we are searching for. Moreover, iterate $x_{k-1}$ is denoted by $z$ and $x_{k}$ is denoted by $x$, for the sake of simplicity.

In the following we recall some basic dynamical concepts (see [9,23]).

Definition 1. If a fixed point $(z, x)$ of operator $G$ is different from $(r, r)$, where $r$ is a zero of $f$, it is called strange fixed point.

Definition 2. Let $G: \mathbb{R}^{2} \rightarrow \mathbb{R}^{2}$ be a vectorial function. The orbit of a point $\bar{x} \in \mathbb{R}^{2}$ is defined as the set of successive images of $\bar{x}$ by the vectorial function, $\left\{\bar{x}, G(\bar{x}), \ldots, G^{m}(\bar{x}), \ldots\right\}$.

The dynamical behavior of the orbit of a point of $\mathbb{R}^{2}$ is classified depending on its asymptotical behavior.

Definition 3. A point $x^{*} \in \mathbb{R}^{2}$ is a k-periodic point if $G^{k}\left(x^{*}\right)=x^{*}$ and $G^{p}\left(x^{*}\right) \neq x^{*}$, for $p=1,2, \ldots, k-1$.

The stability of fixed points for multivariable nonlinear operators, see for example [23], satisfies the following statements:

Theorem 2. Let $G$ from $\mathbb{R}^{n}$ to $\mathbb{R}^{n}$ be $\mathcal{C}^{2}$. Assume $x^{*}$ is a k-periodic point. Let $\lambda_{1}, \lambda_{2}, \ldots, \lambda_{n}$ be the eigenvalues of $G^{\prime}\left(x^{*}\right)$.

a) If all the eigenvalues $\lambda_{j}$ have $\left|\lambda_{j}\right|<1$, then $x^{*}$ is attracting.

b) If one eigenvalue $\lambda_{j_{0}}$ has $\left|\lambda_{j_{0}}\right|>1$, then $x^{*}$ is unstable, that is, repelling or saddle.

c) If all the eigenvalues $\lambda_{j}$ have $\left|\lambda_{j}\right|>1$, then $x^{*}$ is repelling.

In addition, a fixed point is called hyperbolic if all the eigenvalues $\lambda_{j}$ of $G^{\prime}\left(x^{*}\right)$ have $\left|\lambda_{j}\right| \neq 1$. In particular, if there exist an eigenvalue $\lambda_{i}$ such that $\left|\lambda_{i}\right|<1$ and an eigenvalue $\lambda_{j}$ such that $\left|\lambda_{j}\right|>1$, the hyperbolic point is called saddle point.

Then, if $x^{*}$ is an attracting fixed point of function $G$, its basin of attraction $\mathcal{A}\left(x^{*}\right)$ is defined as the set of pre-images of any order such that

$$
\mathcal{A}\left(x^{*}\right)=\left\{x^{(0)} \in \mathbb{R}^{2}: G^{m}\left(x^{(0)}\right) \rightarrow x^{*}, m \rightarrow \infty\right\}
$$

where $x^{(0)}=\left(z_{0}, x_{0}\right) \in \mathbb{R}^{2}$.

The set of the different basins of attraction define the dynamical plane of the system in $(z, x)$-space. The dynamical plane of a method is built by iterating a mesh of points and painting them in different colors depending on the attractor they converge to. In our study we consider the quadratic polynomials $p_{1}(x)=x^{2}-1$, $p_{2}(x)=x^{2}+1, p_{3}(x)=x^{2}$ and also the cubic polynomials $p_{4}(x)=x^{3}-x, p_{5}(x)=x^{3}+x, p_{6}(x)=x^{3}$ and $p_{7}(x)=x^{3}+\gamma x+1$, where $\gamma$ is a real parameter. We use these polynomials as any quadratic or cubic one can be derived as a combination of those. We are going to analyze the fixed points (and their stability) of the multidimensional rational functions associated to the proposed family on these polynomials. We also calculate bifurcations diagrams and dynamical planes for different members of the family that confirm the theoretical results.

\subsection{Behavior on quadratic polynomials}

Let us consider $p_{1}(x)=x^{2}-1$; in this case, the operator of our family is

$$
O_{p_{1}, \beta}(z, x)=\left(x, \frac{1+\beta x^{2}}{x+\beta x}\right),
$$


and its Jacobian matrix is

$$
O_{p_{1}, \beta}^{\prime}(z, x)=\left(\begin{array}{cc}
0 & 1 \\
0 & \frac{-1+\beta x^{2}}{(1+\beta) x^{2}}
\end{array}\right) .
$$

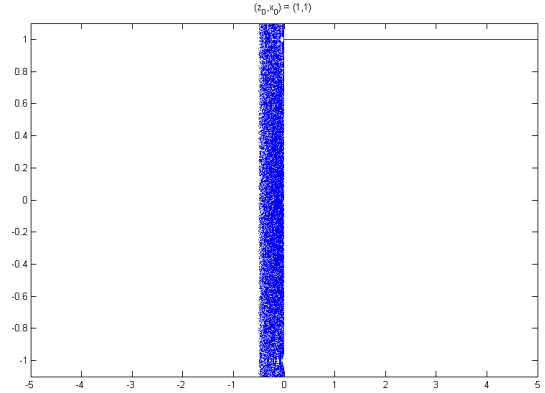

(a) $x^{(0)}=(1,1)$

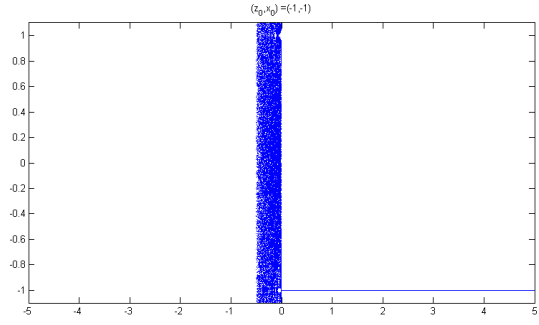

(b) $x^{(0)}=(-1,-1)$

Fig. 1: Bifurcation diagrams of family $O_{p_{1}}(z, x, \beta)$

It is easy to observe that the fixed points of this operator are $(1,1)$ and $(-1,-1)$, that correspond to the roots of $p_{1}(x)$. So, there are no strange fixed points. In order to analyze the stability of these fixed points, we calculate the eigenvalues of $O_{p_{1}, \beta}^{\prime}(1,1)$ and $O_{p_{1}, \beta}^{\prime}(-1,-1)$. In both cases, the eigenvalues are the same: $\lambda_{1}=0$ and $\lambda_{2}=\frac{-1+\beta}{1+\beta}$. It is clear that, if $\beta>0,\left|\lambda_{i}\right|<1 i=1,2$ and points $(1,1)$ and $(-1,-1)$ are attractive. In other case, $\beta<0$, the fixed points are saddle points. Let us remark that this a behavior that differs from the standard one of iterative methods without memory, as those which posses second order of convergence satisfy that the searched simple roots $\alpha$ of the nonlinear functions are always attracting (in fact, they are superattracting, that is $\left.f^{\prime}(\alpha)=0\right)$.



(a) $\beta=0.8$

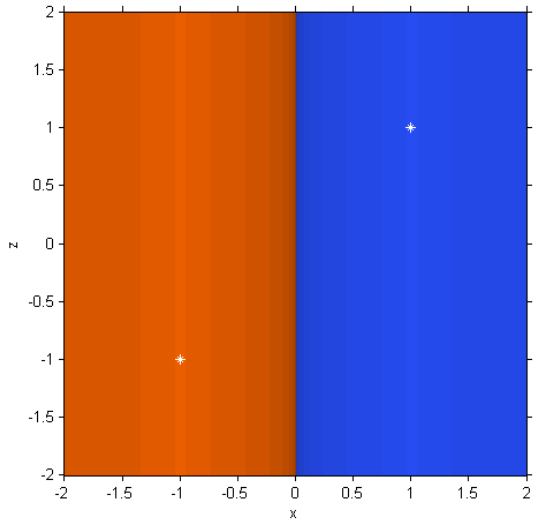

(b) $\beta=1$

Fig. 2: Dynamical planes of family $O_{p_{1}, \beta}(z, x)$

In order to deep in the analysis of the whole parametric family, it is frequent to use a bifurcation diagram, called Feigenbaum diagram ( [18]), to study the changes of behavior of a fixed point, depending on the values of parameter $\beta$. In these diagrams, the abscissas coordinates correspond to values of $\beta$ and the ordinates are associated to the iterations $x$. By using a fixed point as initial guess, for each value of $\beta$, 500 iterations are performed although only the last 300 are plotted. These bifurcations can show a change of stability of the fixed points, or even doubling-period bifurcations (where the periodic or fixed point splits into a periodic orbit with 
a period that duplicates the previous one), pitch-fork bifurcations (where the fixed point changes the stability and also appears a periodic orbit which holds the previous stability of the fixed points), etc. Summarizing, these kind of diagrams show us the way from regularity to chaos, if it exists.

In Figures $1 \mathrm{a}$ and $1 \mathrm{~b}$ it can be observed that, for $\beta>0$, the processes converge to the roots, meanwhile it is observed chaotic behavior for $-\frac{1}{2}<\beta<0$. Some of these behavior are visualized in Figures $2 \mathrm{a}$ and $2 \mathrm{~b}$, where a mesh of $400 \times 400$ initial estimations $x^{(0)}=(z, x)$ is made. For each starting point, a maximum of 40 iterations is performed, with the stopping criterium of distance to the root of $10^{-3}$. When the orbit of an initial estimation is close to one fixed point, it is plotted in a color assigned to this point; the color is brighter as quicker is the convergence.

If $p_{2}(x)$ is considered, the associated operator is

$$
O_{p_{2}, \beta}(z, x)=\left(x, \frac{-1+\beta x^{2}}{(1+\beta) x}\right)
$$

and its Jacobian matrix is

$$
O_{p_{2}, \beta}^{\prime}(z, x)=\left(\begin{array}{cc}
0 & 1 \\
0 & \frac{1+\beta x^{2}}{(1+\beta) x^{2}}
\end{array}\right) .
$$

Let us remark that as $p_{2}(x)$ has not real roots. Moreover, $O p_{2}, \beta(z, x)$ has not strange fixed points. So, its

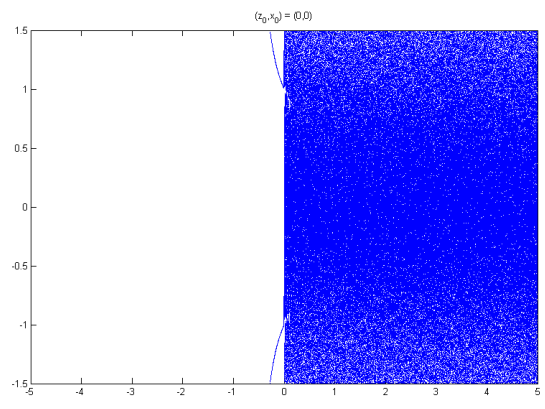

(a) $O_{p_{2}, \beta}(0,0)$

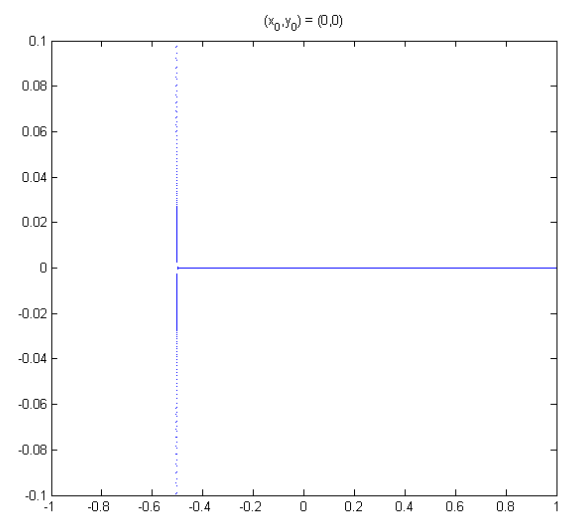

(b) $O_{p_{3}, \beta}(0,0)$

Fig. 3: Bifurcation diagrams starting from $(0,0)$

dynamics is erratic. In Figure 3a, the bifurcation diagram is presented for $O_{p_{2}}(z, x, \beta)$, being the starting point $(0,0)$. As in this case there are nor real roots nor real strange fixed points, it is observed as trajectories are dense for $\beta>0$.

Moreover, small stable trajectories are observed for values of $\beta$ close to zero. In Figure 4 , the limit trajectories of $O_{p_{2}, \beta}(z, x)$ are plotted for $(-1.0392,-1.0392)$ as a starting point and $\beta=-0.0371$. In this picture, 1000 iterations of the operator have been calculated; the first hundred are omitted and from $n=101$ to $n=200$ are plotted in blue, meanwhile they are magenta circles from $n=201$ until $n=1000$. It can be observed as there exist to symmetric periodic attractors, whose detail can be observed in Figure 4b, that are extremely small.

Finally, for $p_{3}(x)=x^{2}$, the associated operator is

$$
O_{p_{3}, \beta}(z, x)=\left(x, \frac{\beta x}{1+\beta}\right),
$$

and its Jacobian matrix is

$$
O_{p_{3}, \beta}^{\prime}(z, x)=\left(\begin{array}{cc}
0 & 1 \\
0 & \frac{\beta}{1+\beta}
\end{array}\right) .
$$




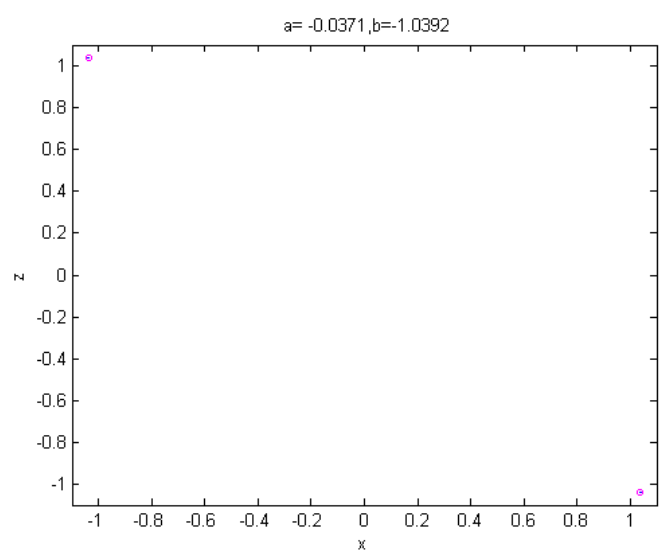

(a) Symmetric attractors

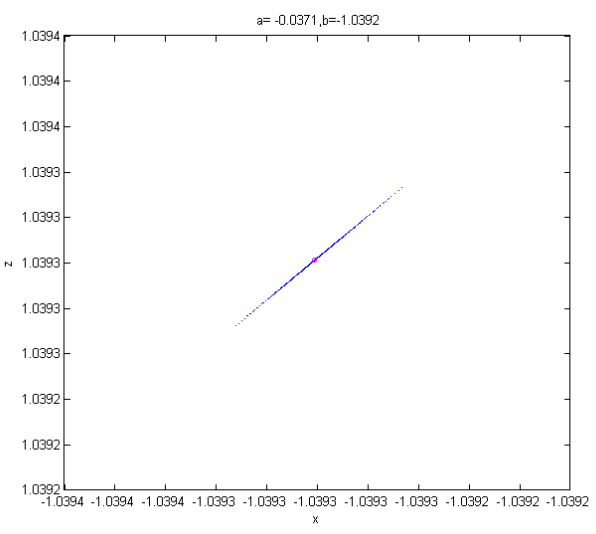

(b) A detail

Fig. 4: Periodic attractors of $O_{p_{2}, \beta}(-1.0392,-1.0392)$ for $\beta=-0.0371$

In this case, the only fixed point is $(0,0)$ and the eigenvalues of $O_{p_{3}, \beta}^{\prime}(0,0)$ are $\lambda_{1}=0$ and $\lambda_{2}=\frac{\beta}{1+\beta}$. So, $(0,0)$ is an attracting point for $\beta>-\frac{1}{2}$. In case of the bifurcation diagram of $O_{p_{3}, \beta}(0,0)$, see Figure $3 \mathrm{~b}$, divergence is observed for values close but lower to $\beta=-0.5$, and stable behavior for higher values of parameter $\beta$.

In Figures $5 \mathrm{a}$ and $5 \mathrm{~b}$ two dynamical planes are showed for different values of parameter $\beta$; it is observed that convergence to the only fixed point is much quicker for values close to zero than for Kurchatov's method.

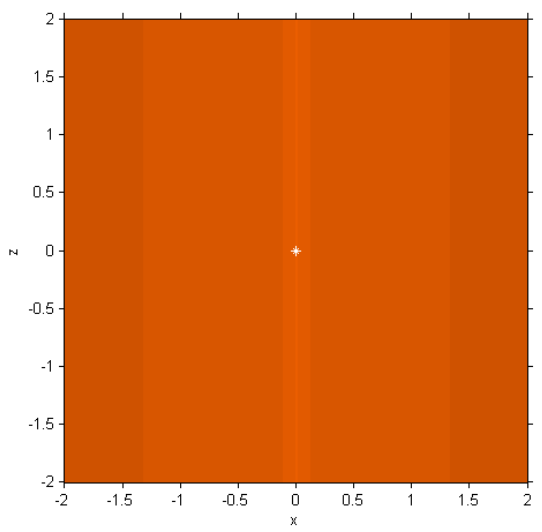

(a) $\beta=0.1$

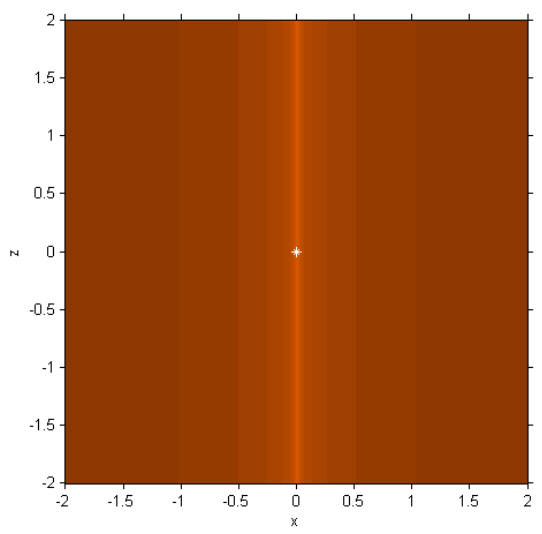

(b) $\beta=1$

Fig. 5: Dynamical planes of family $O_{p_{3}, \beta}(z, x)$

Let us remark that the behavior of family (7) on quadratic polynomials is similar to that of iterative methods without memory, as the previous iterate $z$ does not appear in the analytical expression of its associated operators.

\subsection{Behavior on cubic polynomials}

We analyze now the behavior of members of family (7) on cubic polynomials, that is, on $p_{i}(x), i=4,5,6,7$. For each one, we will calculate the fixed points of the respective fixed point operators, analyze their stability and study possible chaotic performances. 


\subsubsection{Rational function associated to the proposed family on $p_{4}(x)=x^{3}-x$}

We denote by $O_{p_{4}, \beta}(z, x)$ the rational function associated to the proposed class on polynomial $p_{4}(x)$. Its expression is

$$
O_{p_{4}, \beta}(z, x)=\left(x, x+\frac{x-x^{3}}{-1+(\beta+1)^{2} x^{2}-\beta(1+\beta) x z+\beta^{2} z^{2}}\right),
$$

being its Jacobian matrix

$O_{p_{4}, \beta}^{\prime}(z, x)=\left(\begin{array}{cc}0 & 1 \\ -\frac{\beta x\left(-1+x^{2}\right)(x+\beta x-2 \beta z)}{\left(-1+(1+\beta)^{2} x^{2}-\beta(1+\beta) x z+\beta^{2} z^{2}\right)^{2}} & 1+\frac{(1+\beta) x\left(-1+x^{2}\right)(2(1+\beta) x-\beta z)}{\left(-1+(1+\beta)^{2} x^{2}-\beta(1+\beta) x z+\beta^{2} z^{2}\right)^{2}}+\frac{1-3 x^{2}}{-1+(1+\beta)^{2} x^{2}-\beta(1+\beta) x z+\beta^{2} z^{2}}\end{array}\right)$.

The number of fixed points of $O_{p_{4}, \beta}(z, x)$ depends on the value of $\beta$. In the following result we summarize this fact.

Proposition 1. By analyzing the equation $O_{p_{4}, \beta}(z, x)=(z, x)$, we have the following statements:

a) There not exist strange fixed points.

b) For all $\beta \neq-1$, the fixed points are $(-1,-1),(0,0)$ and $(1,1)$, corresponding to the roots of $p_{4}(x)$.

c) If $\beta=-1$, the unique fixed point is $(0,0)$.

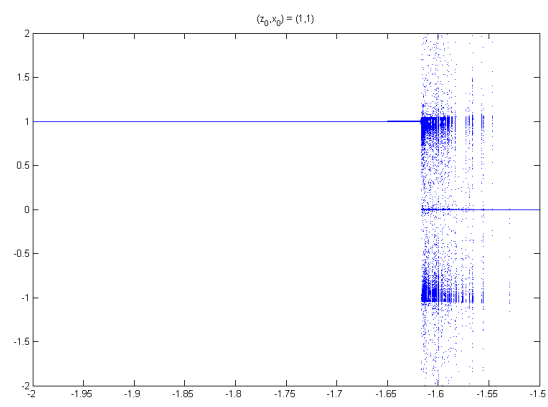

(a) $\beta$ close to $\frac{-1-\sqrt{5}}{2}$

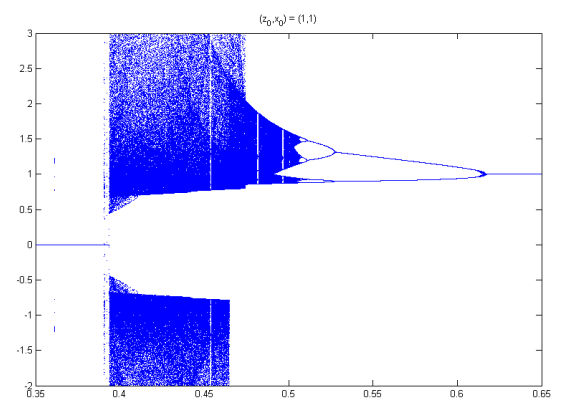

(b) $\beta$ close to $\frac{-1+\sqrt{5}}{2}$

Fig. 6: Bifurcation diagrams of family $O_{p_{4}}(z, x, \beta)$

Respect to the stability of these fixed points, we can establish the following result.

Proposition 2. Regarding the stability of fixed points of operator $O_{p_{4}, \beta}(z, x)=(z, x)$ :

a) Fixed point $(0,0)$ is always attracting.

b) Fixed points $(-1,-1)$ and $(1,1)$ are simultaneously attracting if and only if $\beta \in]-\infty, \frac{-1-\sqrt{5}}{2}[\cup$ ]$\frac{-1+\sqrt{5}}{2},+\infty[$.

Proof: The results are straightforward from the analysis of the eigenvalues of $O_{p_{4}, \beta}^{\prime}(z, x)$ at these points.

a) The eigenvalues of matrix

$$
O_{p_{4}, \beta}^{\prime}(0,0)=\left(\begin{array}{ll}
0 & 1 \\
0 & 0
\end{array}\right) .
$$

are $\lambda_{1}=\lambda_{2}=0$. So, fixed point $(0,0)$ is attracting and this fact does not depend on $\beta$. 
b) Firstly, we note that

$$
O_{p_{4}, \beta}^{\prime}(1,1)=O_{p_{4}}^{\prime}(-1,-1, \beta)=\left(\begin{array}{cc}
0 & 1 \\
0 & \frac{-2+\beta+\beta^{2}}{\beta(1+\beta)}
\end{array}\right) .
$$

Therefore, both points $(-1,-1)$ and $(1,1)$ have the same stability. It is clear that $\lambda_{1}=0$ and $\lambda_{2}=$ $\left(-2+\beta+\beta^{2}\right) /(\beta(1+\beta))$. So, they are simultaneously attracting if and only if $\left|\lambda_{2}\right|<1$, that is, if $\beta<\frac{-1-\sqrt{5}}{2}$ or $\beta>\frac{-1+\sqrt{5}}{2}$, and the proof is finished.

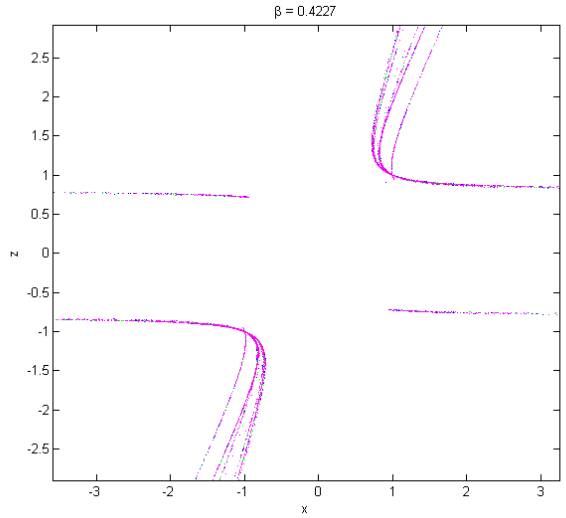

(a) $\beta=0.4227$

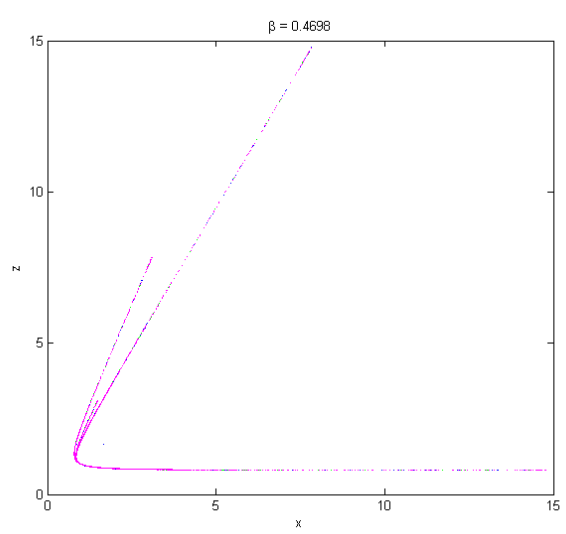

(c) $\beta=0.4698$

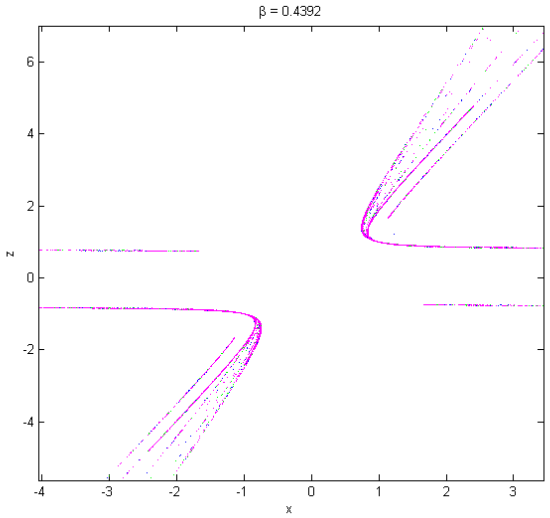

(b) $\beta=0.4392$

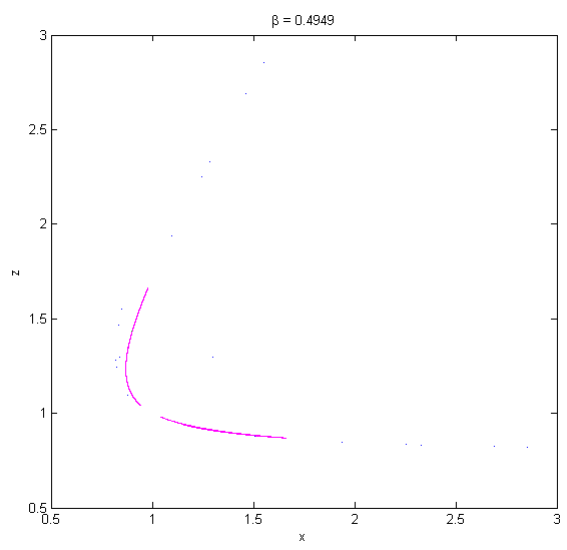

(d) $\beta=0.4949$

Fig. 7: Strange attractors in the orbit of $O_{p_{4}, \beta}(z, x)$

As there not exist strange fixed points, unstable behavior can be found when fixed points change their stability. This is observed in the bifurcation diagrams (see Figure 6 ), where $(1,1)$ has been used as initial point and a typical graph of period-doubling bifurcation is observed for values of $\beta \in\left[0.35, \frac{-1+\sqrt{5}}{2}[\right.$. In Figure $6 \mathrm{a}$, it can be observed that $(1,1)$ converges to $(0,0)$ when $\beta$ is higher than but close to $\frac{-1+\sqrt{5}}{2}$. On the other hand, from the upper bound of $\beta$ to the left (values of $\beta$ close but lower than $\frac{-1+\sqrt{5}}{2}$, see Figure $6 \mathrm{~b}$ ), the attracting fixed point $(1,1)$ bifurcates in a 2-periodic orbit, that yields a 4-periodic orbit and, further on, 
results an strange attractor [24], as can be observed in Figure 7, where the limit orbits of the rational function $O_{p_{4}, \beta}(z, x)$ can be observed for close values of parameter $\beta$.

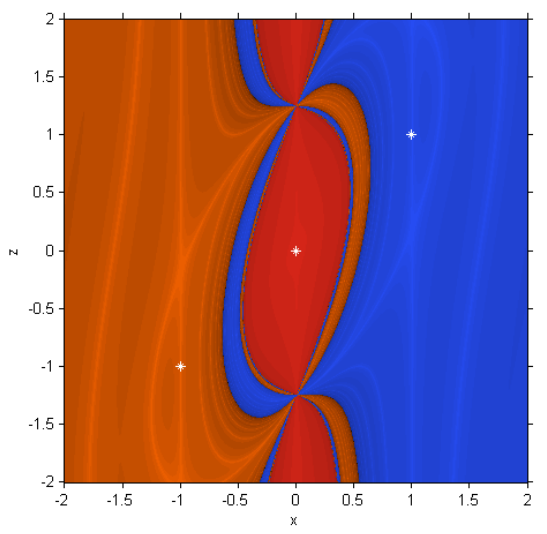

(a) $\beta=0.8$

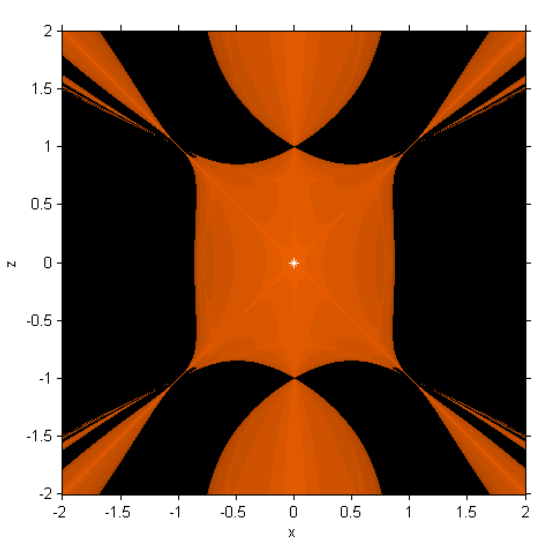

(c) $\beta=-1$

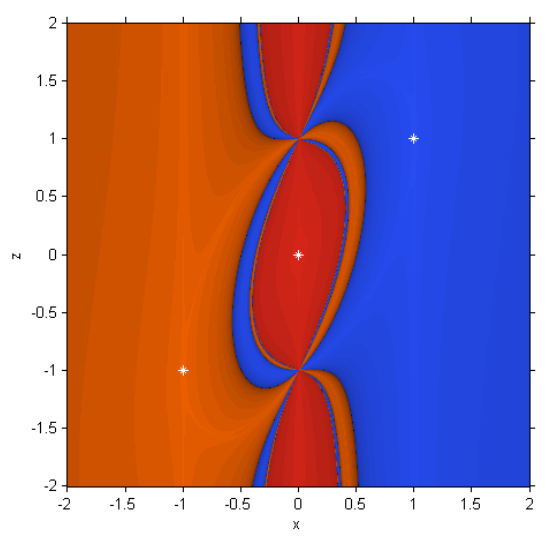

(b) $\beta=1$

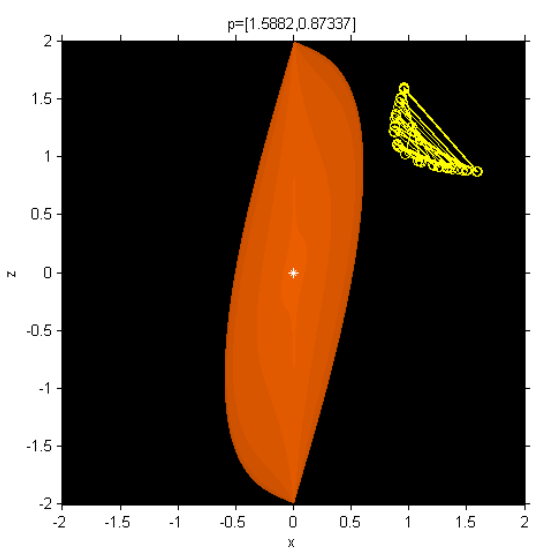

(d) $\beta=0.5$

Fig. 8: Dynamical planes of $O_{p_{4}, \beta}(z, x)$

In Figure 5, some dynamical planes are presented: two stable cases (Figures 8a and 8b) and another two corresponding to unstable behavior (Figures $8 \mathrm{c}$ and $8 \mathrm{~d}$ ). In the first two cases, wide basins of attraction of the three roots appear; in case $\beta=-1$, the only fixed point is $(0,0)$ in spite of the polynomial has three real roots. Finally, in case $\beta=0.5$, there are three fixed points but only $(0,0)$ is attracting. Moreover, it is observed the effect of the strange attractor marked in yellow color. This strange attractor is that obtained in Figure 7.

\subsubsection{Rational function associated to the proposed family on $p_{5}(x)=x^{3}+x$}

Now, let us denote by $O_{p_{5}, \beta}(z, x)$ the rational function associated to family $(7)$ on polynomial $p_{5}(x)$. Its expression is

$$
O_{p_{5}, \beta}(z, x)=\left(x, x-\frac{x+x^{3}}{1+(\beta+1)^{2} x^{2}-\beta(1+\beta) x z+\beta^{2} z^{2}}\right),
$$

being its Jacobian matrix

$$
O_{p_{5}, \beta}^{\prime}(z, x)=\left(\begin{array}{cc}
0 & 1 \\
-\frac{\beta\left(x+x^{3}\right)(x+\beta x-2 \beta z)}{\left(1+(1+\beta)^{2} x^{2}-\beta(1+\beta) x z+\beta^{2} z^{2}\right)^{2}} & 1+\frac{-1-(1+\beta)^{2} x^{4}+2 \beta(1+\beta) x^{3} z-\beta^{2} z^{2}+x^{2}\left(-2+\beta\left(2+\beta-3 \beta z^{2}\right)\right)}{\left(1+(1+\beta)^{2} x^{2}-\beta(1+\beta) x z+\beta^{2} z^{2}\right)^{2}}
\end{array}\right) .
$$


Now, by analyzing the equation $O_{p_{5}, \beta}(z, x)=(z, x)$, we conclude that the only real fixed point is $(0,0)$ and it is attracting as the eigenvalues of matrix $O_{p_{5}, \beta}^{\prime}(0,0)$ are $\lambda_{1}=\lambda_{2}=0$, with independence of the value of $\beta$. In Figure 9, the dynamical planes associated to operator $O_{p_{5}, \beta}(z, x)=(z, x)$ can be observed for $\beta=1$ and $\beta=0.1$; let us remark that although the basin of attraction of the only fixed point $(0,0)$ is very wide in both cases, the brighter color of Figure 9a show us that the speed of convergence in the case of $\beta$ close to zero is greater than the one of Kurchatov's method.

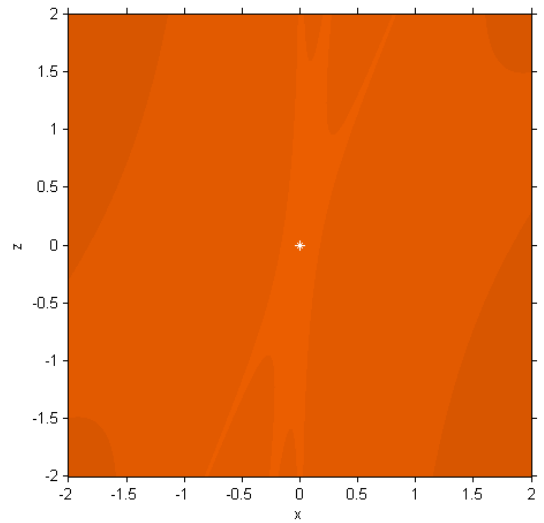

(a) $\beta=0.1$

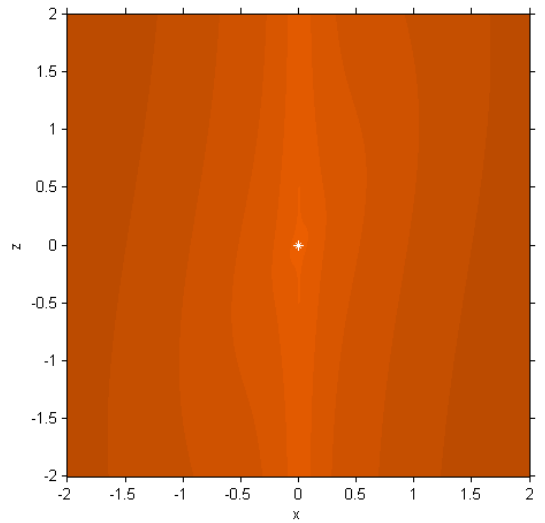

(b) $\beta=1$

Fig. 9: Dynamical planes of $O_{p_{5}, \beta}(z, x)$

\subsection{Rational function associated to the proposed family on $p_{6}(x)=x^{3}$}

In this case, we denote by $O_{p_{6}}(z, x, \beta)$ the rational function associated to family $(7)$ on polynomial $p_{6}(x)$. Its expression is

$$
O_{p_{6}, \beta}(z, x)=\left(x, x-\frac{x^{3}}{(\beta+1)^{2} x^{2}-\beta(1+\beta) x z+\beta^{2} z^{2}}\right),
$$

being its Jacobian matrix

$$
O_{p_{6}, \beta}^{\prime}(z, x)=\left(\begin{array}{cc}
0 & 1 \\
-\frac{\beta x^{3}(x+\beta x-2 \beta z)}{\left((1+\beta)^{2} x^{2}-\beta(1+\beta) x z+\beta^{2} z^{2}\right)^{2}} & 1+\frac{(1+\beta) x^{3}(2(1+\beta) x-\beta z)}{\left((1+\beta)^{2} x^{2}-\beta(1+\beta) x z+\beta^{2} z^{2}\right)^{2}}-\frac{3 x^{2}}{(1+\beta)^{2} x^{2}-\beta(1+\beta) x z+\beta^{2} z^{2}}
\end{array}\right) .
$$

Again, we conclude that the only real fixed point is $(0,0)$ but its stability can not be determined by Theorem 2 as the Jacobian matrix is not defined at $(0,0)$. As $(0,0)$ corresponds to a multiple root, the convergence of the family is only linear. Due to this fact, dynamical planes need more iterations in order to observe the basins of attraction. Dynamical planes of Figure 10 have been obtained by using a maximum number of iterations of 80. In them, it can be observed that $(0,0)$ behaves as a saddle point for values of $\beta \in[-1.2,-0.211[$ (see Figure 10b). Out of this interval $(0,0)$ behaves as an attracting fixed point with global convergence, as in case of Kurchatov's method (Figure 10d); moreover, the speed of convergence is higher for values of $\beta$ close to zero, as can be observed in Figures 10b and 10c.

\subsection{Rational function associated to the proposed family on $p_{7}(x)=x^{3}+\gamma x+1$}

The rational function associated to family $(7)$ on $p_{7}(x)$ has the following expression

$$
O_{p_{7}, \beta, \gamma}(z, x)=\left(x, x-\frac{x^{3}+\gamma x+1}{\gamma+(\beta+1) x^{2}-\beta(1+\beta) x z+\beta^{2} z^{2}}\right) .
$$




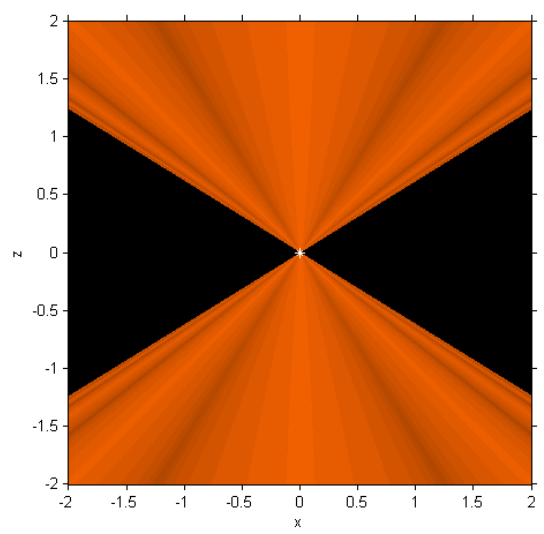

(a) $\beta=-1$

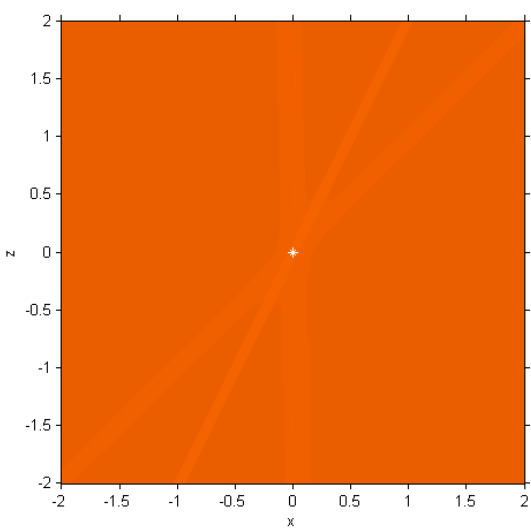

(c) $\beta=0.01$

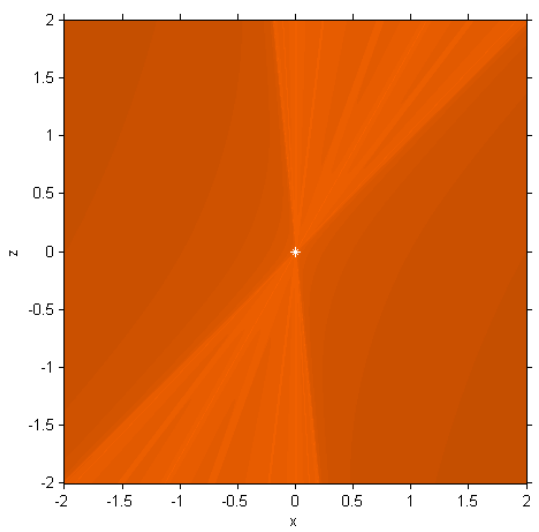

(b) $\beta=-0.1$

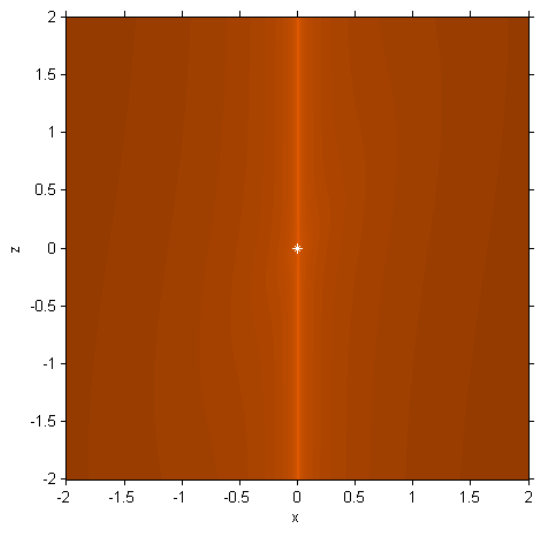

(d) $\beta=1$

Fig. 10: Dynamical planes of $O_{p_{6}}(z, x, \beta)$

Its associated Jacobian matrix is

$O_{p_{7}, \beta, \gamma}^{\prime}(z, x)=\left(\begin{array}{cc}0 & 1 \\ -\frac{\beta\left(1+\gamma x+x^{3}\right)(x+\beta x-2 \beta z)}{\left(\gamma+(1+\beta)^{2} x^{2}-\beta(1+\beta) x z+\beta^{2} z^{2}\right)^{2}} & 1+\frac{(1+\beta)\left(1+\gamma x+x^{3}\right)(2(1+\beta) x-\beta z)}{\left(\gamma+(1+\beta)^{2} x^{2}-\beta(1+\beta) x z+\beta^{2} z^{2}\right)^{2}}-\frac{\gamma+3 x^{2}}{\gamma+(1+\beta)^{2} x^{2}-\beta(1+\beta) x z+\beta^{2} z^{2}}\end{array}\right)$.

The complexity of calculations is high, due to the existence of two parameters in the fixed point operator. So, the following statements have been checked by using the symbolic package of Mathematica.

We affirm the following statements on the fixed points of $O_{p_{7}, \beta, \gamma}(z, x)$ :

- The only fixed points of $O_{p_{7}, \beta, \gamma}(z, x)$ are the roots of this polynomial, $r_{i}, i=1,2,3$.

- When these roots are real? It can be checked that, for any $\beta \notin\{-2,-1,-1 / 2,1\}$ :

- If $\gamma<\gamma_{0}$ and $\gamma \neq \gamma_{1}, r_{1}, r_{2}$ and $r_{3}$ are real.

- If $\gamma>\gamma_{0}, p_{7}(x)$ has only a real root.

- If $\gamma=\gamma_{0}$ or $\gamma=\gamma_{1}$, the roots of $p_{7}(x)$ are real and one of them is double,

where $\gamma_{0}$ is the real root of $s(t)=27+4 t^{4}, \gamma_{0} \approx-1.889881575$ and $\gamma_{1}$ is the real root of polynomial $q(t)=1+3 \beta+6 \beta^{2}+7 \beta^{3}+6 \beta^{4}+3 \beta^{5}+\beta^{6}+\left(\beta^{2}+2 \beta^{3}+\beta^{4}\right) t^{3}$. 
- For values of $\beta \in\{-2,-1,-1 / 2,1\}$,

- If $\gamma<\gamma_{0} r_{1}, r_{2}$ and $r_{3}$ are real.

- If $\gamma>\gamma_{0}, p_{7}(x)$ has only a real root.

- If $\gamma=\gamma_{0}$, there is only a real root, except in case $\beta=-1$ where roots of $p_{7}(x)$ are real and one of them is double.

In order to analyze the stability of $r_{i}, i=1,2,3$, the eigenvalues of $O_{p_{7}, \beta, \gamma}^{\prime}(z, x)$ must be calculated. For all $\beta, \gamma \in \mathbb{R}, \operatorname{det}\left(O_{p_{7}, \beta, \gamma}^{\prime}\left(r_{i}, r_{i}\right)\right)=0, i=1,2,3$. So, one of the associated eigenvalues is always zero. The second eigenvalue $\lambda_{2}$ depends on $\beta$ and $\gamma$,

$$
\lambda_{2}(\beta, \gamma)=-\frac{(2+\beta) r_{i}\left(-1+\beta^{3}+\beta\left(-1+\beta^{2}\right) \gamma r_{i}\right)}{\left(\gamma+\left(1+\beta+\beta^{2}\right) r_{i}^{2}\right)^{2}}, \quad i=1,2,3 .
$$

Function $\left|\lambda_{2}(\beta, \gamma)\right|$ is called stability function of fixed point $r_{i}, i=1,2,3$, as its asymptotic behavior depends on it.

- For $r_{1}$, when $\left.\left.\beta \in\right]-\infty, \frac{-1-\sqrt{5}}{2}\right] \cup\left[\frac{-1+\sqrt{5}}{2},+\infty\left[\right.\right.$, then $\left|\lambda_{2}(\beta, \gamma)\right|<1$ with independence of $\gamma$. So, $r_{1}$ is attracting in this domain of parameters. For $\beta \in] \frac{-1-\sqrt{5}}{2}, \frac{-1+\sqrt{5}}{2}$ [, we need that $\gamma>\gamma_{2}$ in order to assure that $\left|\lambda_{2}(\beta, \gamma)\right|<1$, where $\gamma_{2}$ is the real root of polynomial $r(t)=-1+6 \beta-6 \beta^{2}-16 \beta^{3}+12 \beta^{4}+24 \beta^{5}+$ $8 \beta^{6}+\left(4-8 \beta-4 \beta^{2}+8 \beta^{3}+4 \beta^{4}\right) t^{3}$. In other case, $r_{1}$ is a saddle point. In Figure 11, we represent $\left|\lambda_{2}(\beta, \gamma)\right|$ in $[-5,5] \times[-6,6]$.

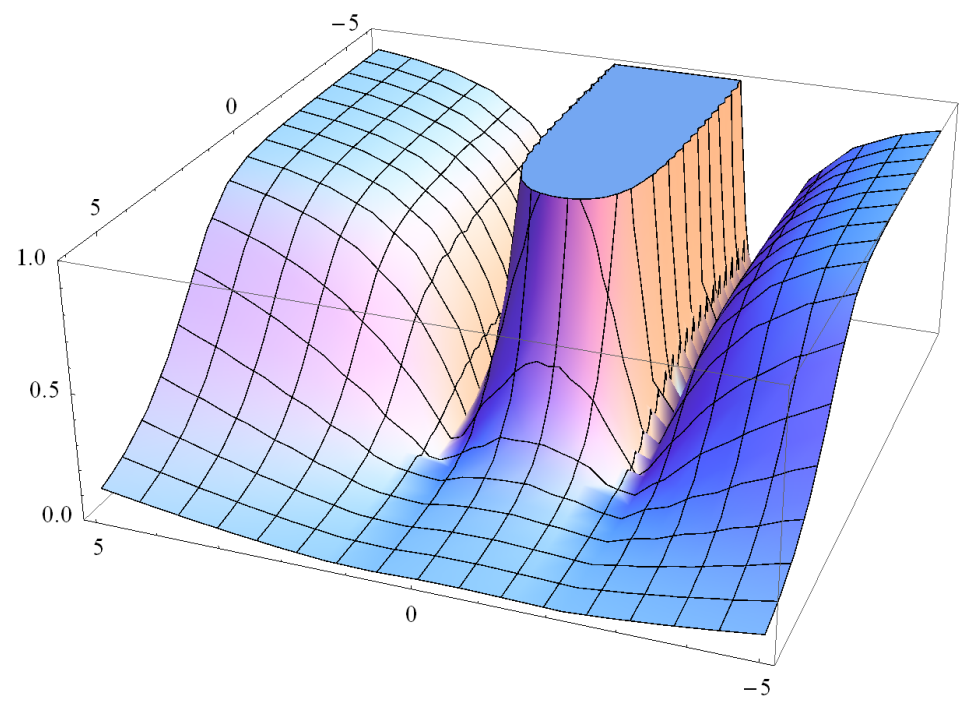

Fig. 11: Stability function $\left|\lambda_{2}(\beta, \gamma)\right|$ for the fixed point $\left(r_{1}, r_{1}\right)$

- Respect to the root $r_{2}$, when $\left.\left.\left.\beta \in\right]-\infty,-2\right] \cup\right] 1,+\infty\left[\right.$ and $\gamma<\gamma_{2}$, then $\left|\lambda_{2}(\beta, \gamma)\right|<1$. For $\left.\left.\beta \in\right]-2,1\right]$ and $\gamma<\gamma_{0},\left|\lambda_{2}(\beta, \gamma)\right|<1$. In both cases $r_{2}$ is an attracting fixed point and it is a saddle point in other cases. This behavior can be observed in Figure 12.

- For $r_{3}$, if $\left.\left.\left.\beta \in\right]-\infty,-2\right] \cup\right] 1,+\infty\left[\right.$, then $\left|\lambda_{2}(\beta, \gamma)\right|<1$ for any value of $\gamma$. However, if $\left.\left.\beta \in\right]-2, \frac{-1-\sqrt{5}}{2}[\cup] \frac{-1+\sqrt{5}}{2}, 1\right]$ and $\gamma<\gamma_{2}$, then $\left|\lambda_{2}(\beta, \gamma)\right|<1$. So, in this domain $r_{3}$ is attracting and if $\beta \in\left[\frac{-1-\sqrt{5}}{2}, \frac{-1+\sqrt{5}}{2}\right]$ or if it does not satisfies any of the previous conditions, then $r_{3}$ is a saddle point (see Figure 13).

For summarizing, the values of $\beta$ where the behavior of the elements of (7) is unstable (not even the roots of $p_{7}(x)$ are attracting fixed points) are those belonging to the interval $\left[\frac{-1-\sqrt{5}}{2}, \frac{-1+\sqrt{5}}{2}\right]$. 


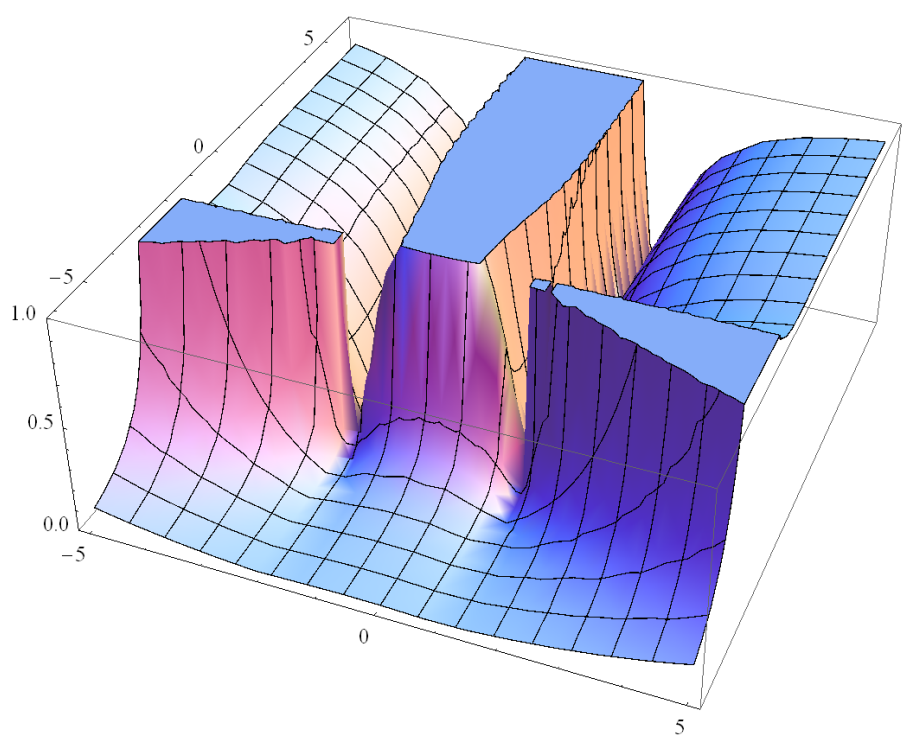

Fig. 12: Stability function $\left|\lambda_{2}(\beta, \gamma)\right|$ for the fixed point $\left(r_{2}, r_{2}\right)$

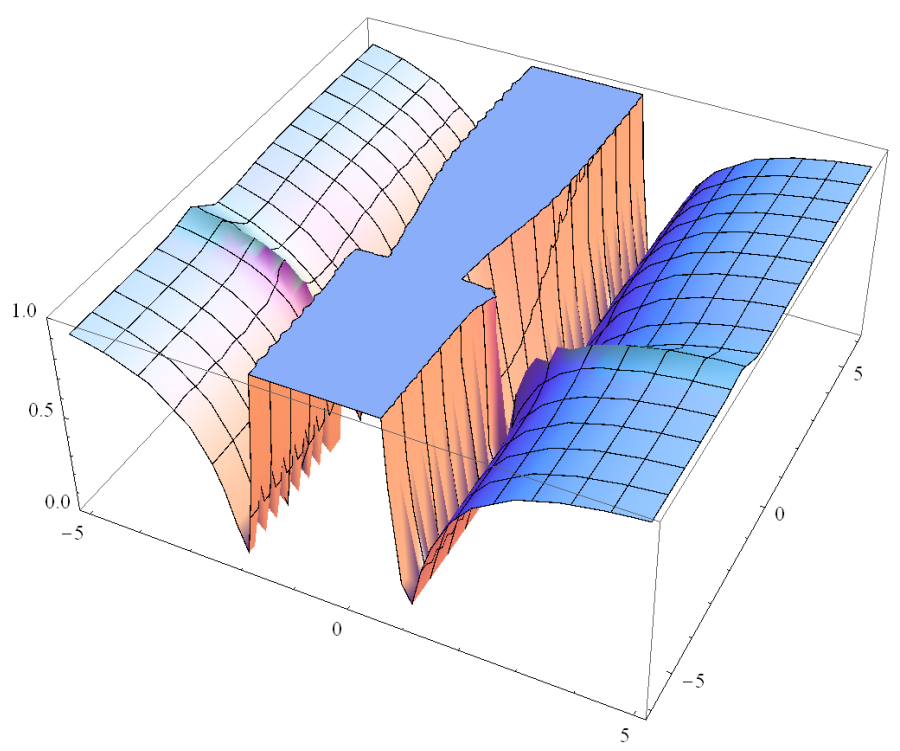

Fig. 13: Stability function $\left|\lambda_{2}(\beta, \gamma)\right|$ for the fixed point $\left(r_{3}, r_{3}\right)$

Some dynamical planes showing the described behavior are presented in Figure 14. In them, we have selected four cases: in Figure 14a, case $\beta=1, \gamma=\gamma_{0}$ is presented, where one of the roots of $p_{7}(x)$ is double; case $14 \mathrm{~b}$ corresponds to values of parameters preserving $r_{i} i=1,2,3$ as simple real roots, meanwhile case $14 \mathrm{c}$ is associated to three simple real roots, but only one of them is attracting (marked with a white star in the figure and the saddle ones are marked as white circles). In Figure 14d, the basin of attraction of the only real root is shown, as it is attractive, being the other ones complex.

Summarizing this dynamical analysis, we have found many elements of the family with a very stable behavior, being some of the even better than Kurchatov's scheme. Under the point of view of the stability, this family is very rich, holding elements with good properties, as well as erratic behavior. 


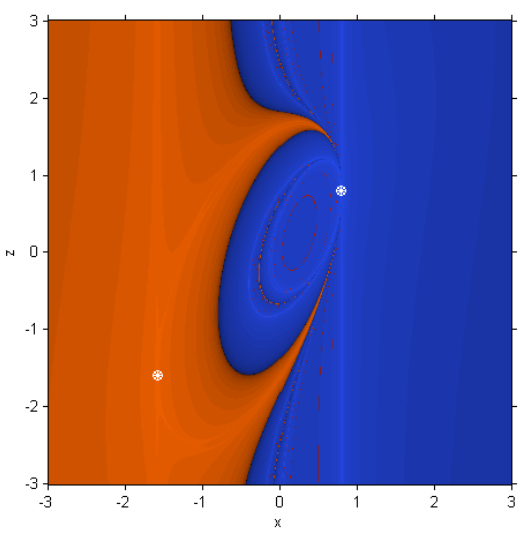

(a) $\beta=1, \gamma=\gamma_{0}$

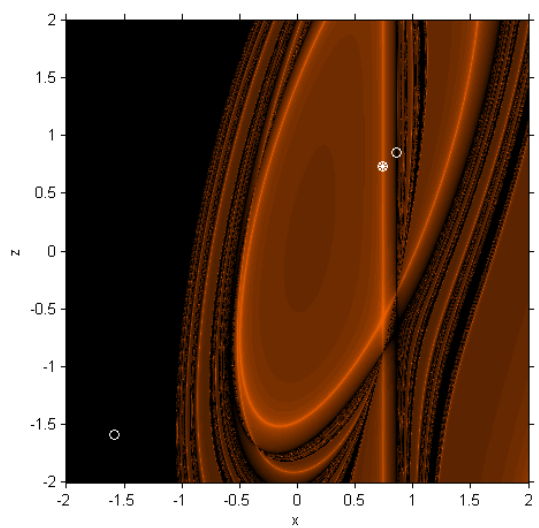

(c) $\beta=0.5, \gamma=-1.9$

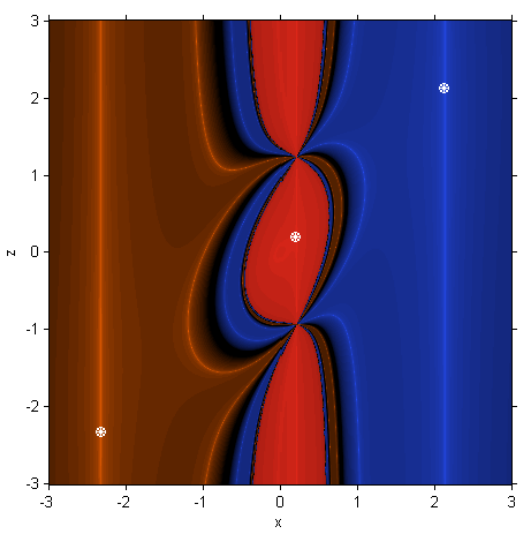

(b) $\beta=2, \gamma=-5$

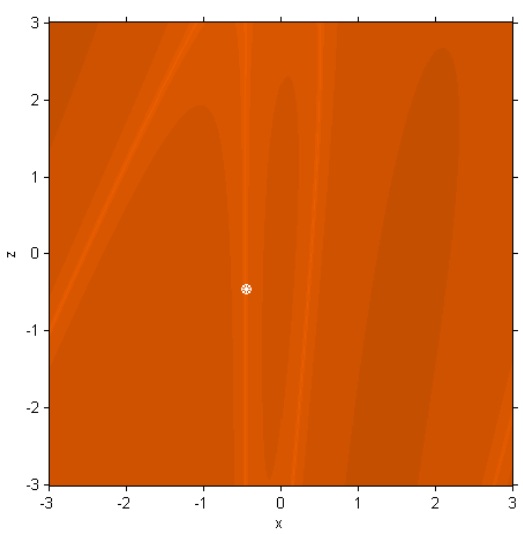

(d) $\beta=0.1, \gamma=2$

Fig. 14: Dynamical planes of $O_{p_{7}}(z, x, \beta)$

\section{Handling nonlinear systems}

\section{Numerical reports}

The main elements which contribute towards the total computational cost are the evaluations of functions, derivatives and inverse operators when dealing with nonlinear systems. 
Example 5.1. We continue with the system $F\left(x_{1}, x_{2}, x_{3}, x_{4}, x_{5}, x_{6}, x_{7}, x_{8}, x_{9}, x_{10}\right)=0$ defined by:

$$
F(x)=\left\{\begin{array}{l}
5 \exp \left(x_{1}-2\right) x_{2}+2 x_{7}{ }^{x_{10}}+8 x_{3}{ }^{x_{4}}-5 x_{6}{ }^{3}-x_{9} \\
5 \tan \left(x_{1}+2\right)+\cos \left(x_{9}{ }^{x_{10}}\right)+x_{2}{ }^{3}+7 x_{3}{ }^{4}-2 \sin ^{3}\left(x_{6}\right) \\
x_{1}{ }^{2}-x_{10} x_{5} x_{6} x_{7} x_{8} x_{9}+\tan \left(x_{2}\right)+2 x_{3}{ }^{x_{4}}-5 x_{6}{ }^{3} \\
2 \tan \left(x_{1}{ }^{2}\right)+2^{x_{2}}+x_{3}{ }^{2}-5 x_{5}{ }^{3}-x_{6}+x_{8}{ }^{\cos \left(x_{9}\right)} \\
10 x_{1}{ }^{2}-x_{10}+\cos \left(x_{2}\right)+x_{3}{ }^{2}-5 x_{6}{ }^{3}-2 x_{8}-4^{x_{9}} \\
\cos ^{-1}\left(x_{1}{ }^{2}\right) \sin \left(x_{2}\right)-2 x_{10} x_{5}{ }^{4} x_{6} x_{9}+x_{3}{ }^{2} \\
x_{1} x_{2}{ }^{x_{7}}-x_{8} x_{10}+x_{3}{ }^{5}-5 x_{5}{ }^{3}+x_{7} \\
\cos ^{-1}\left(-10 x_{10}+x_{8}+x_{9}\right)+x_{4} \sin \left(x_{2}\right)+x_{3}-15 x_{5}{ }^{2}+x_{7}, \\
10 x_{1}+x_{3}{ }^{2}-5 x_{5}{ }^{2}+10 x_{6}{ }^{x_{8}}-\sin \left(x_{7}\right)+2 x_{9} \\
x_{1} \sin \left(x_{2}\right)-2 x_{10} x_{8}+x_{10}-5 x_{6}-10 x_{9}
\end{array}\right.
$$

where $\alpha \simeq(1.3273490437+0.3502924960 i, 1.058599346-1.748724664 i, 1.0276186794-0.0141308051 i, 3.27395$ $0008+0.127828308 i, 0.8318243937+0.0017551949 i,-0.4853245912+0.6848776400 i, 0.1693667630+0.184091$ 7580i,1. $534419958-0.321214766 i, 2.086379651+0.426342755 i,-1.989592331+1.478395393 i)^{*}$, and $x^{(0)}=$ $(1.4+0.5 I, 1.1-2.0 I, 1.0-0.2 I, 2.5+0.5 I, 0.8-0.1 I,-0.4+1 . I, 0.1+0.1 I, 1.4-0.6 I, 2.0+0.5 I,-2.0+1.45 I)^{*}$.

Table 2. Results of comparisons for different methods in Example 5.1.

\begin{tabular}{lllll}
\hline Iterative methods & IT & $R^{(k+1)}$ & $\varrho$ & Time \\
\hline ST1 & & & & \\
ST2 & & & & \\
KM & & & & \\
PM & & & & \\
\hline
\end{tabular}

\section{Concluding remarks}

Moreover, theoretical results concerning order of convergence and computational efficiency have been confirmed in numerical examples.

\section{Acknowledgements}

\section{References}

[1] F. Ahmad, E. Tohidi, J.A. Carrasco, A parameterized multi-step Newton method for solving systems of nonlinear equations, Numerical Algorithms, 71 (2016) 631-653.

[2] W.F.H. Al-Shameri, Dynamical Properties of the Hénon Mapping, Int. J. Math. Anal. 6(49) (2012) 2419-2430.

[3] S. Amat, S. Busquier, S. Plaza, Dynamics of the King and Jarratt iterations, Aeq. Math. 69 (2005), 212-223.

[4] I.K. Argyros, The Secant method and fixed points of nonlinear operators, Monatsh Math 106 (1988), 85-94.

[5] I.K. Argyros, Concerning the semilocal convergence of Newtons method and convex majorants, Rendiconti del Circolo Matematico di Palermo, 57 (2008), 331-341.

[6] B. Campos, A. Cordero, J.R. Torregrosa, P. Vindel, A multidimensional dynamical approach to iterative methods with memory, Applied Mathematics and Computation, 271 (2015), 701-715.

[7] A. Cordero, F. Soleymani, J.R. Torregrosa, S. Shateyi Basins of Attraction for Various Steffensen-Type Methods Journal of Applied Mathematics, 2014 (2014), Article ID 539707.

[8] A. Cordero, J.G. Maimó, J.R. Torregrosa, Maria P. Vassileva, Stability of a fourth order bi-parametric family of iterative methods, Journal of Computational and Applied Mathematics, (2016), DOI: 10.1016/j.cam.2016.01.013.

[9] R. Devaney, An Introduction to Chaotic Dynamical Systems, Addison-Wesley Publ. Co., New York, 1989. 
[10] Y.H. Geum, Y.I. Kim, Á. Alberto Magreñán, A biparametric extension of King's fourth-order methods and their dynamics, Applied Mathematics and Computation, 282 (2016), 254-275.

[11] M. Grau-Sánchez, À. Grau, M. Noguera, Frozen divided difference scheme for solving systems of nonlinear equations, J. Comput. Appl. Math. 235 (2011), 1739-1743.

[12] M. Grau-Sánchez, M. Noguera, S. Amat, On the approximation of derivatives using divided difference operators preserving the local convergence order of iterative methods, J. Comput. Appl. Math., 237 (2013), 363-372.

[13] M. Hénon, A Two-dimensional Mapping with a Strange Attrctor, Commun. Math. Phys. 50 (1976) 69-77.

[14] M.A. Hernández-Verón, M.J. Rubio, On a Newton-Kantorovich-type Iterative Process, Num. Funct. Anal. Opt. 37(1) (2016) $65-79$.

[15] L.O. Jay, A note on Q-order of convergence, BIT, 41 (2001), 422-429.

[16] H.T. Kung, J.F. Traub, Optimal order of one-point and multipoint iteration, J. Assoc. Comput. Math. 21 (1974) 634-651.

[17] V.A. Kurchatov, On a method of linear interpolation for the solution of functional equations, Dokl. Akad. Nauk SSSR (Russian) 198 (1971), 524-526. Translation in Soviet Math. Dokl. 12 (1971), 835-838.

[18] S. Lynch, Dynamical Systems with Applications using Matlab, Birkhäuser Basel, Springer International Publishing, 2014.

[19] B. Neta, C. Chun, M. Scott, Basins of attraction for optimal eighth order methods to find simple roots of nonlinear equations, Applied Mathematics and Computation, 227 (2014), 567-592.

[20] J.M. Ortega, , W.C. Rheinboldt, Iterative Solutions of Nonlinear Equations in Several Variables, Academic Press, New York (1970).

[21] Petković, M.S., Neta, B., Petković, L.D., Džunić, J. (ed.).: Multipoint Methods for Solving Nonlinear Equations. Elsevier (2013)

[22] M.S. Petković, B. Neta, L.D. Petković, J. Džunić, Multipoint methods for solving nonlinear equations: A survey, Appl. Math. Comput., 226 (2014), 635-660.

[23] C. Robinson, An Introduction to Dynamical Systems: Continuous and Discrete, Pearson Prentice Hall, New Jersey, 2004.

[24] C. Robinson, What is a Chaotic attractor?, Qual. Th. Dyn. Syst. 7 (2008) 227-236.

[25] S.M. Shakhno, On a Kurchatov's method of linear interpolation for solving nonlinear equations, Proc. Appl. Math. Mech. 4 (2004), 650-651.

[26] J.R. Sharma, R.K. Guha, P. Gupta, Some efficient derivative free methods with memory for solving nonlinear equations, Appl. Math. Comput. 219 (2012), 699-707.

[27] J.F. Steffensen, Remarks on iteration, Skand. Aktuarietidskr. 16 (1933), 64-72.

[28] J.F. Traub, Iterative Methods for the Solution of Equations, Prentice Hall, New York, 1964.

[29] Wolfram Research, Inc., Mathematica, Version 10.0, Champaign, IL (2014).

[30] Q. Zheng, J. Li, F. Huang, Optimal Steffensen-type families for solving nonlinear equations, Appl. Math. Comput. 217 (2011), 9592-9597. 